\title{
Aulas Digitales en la Educación Superior: Caso México
}

\author{
Anderson Monroy*, Ingrid A. Hernández y Martha Jiménez \\ Instituto Politécnico Nacional, Unidad Profesional Interdisciplinaria de Ingeniería y Ciencias Sociales y \\ Administrativas, Av. Té 950, Iztacalco, Granjas México, 08400 Ciudad de México. \\ (e-mail: anderson_monroy.r74@outlook.com; ingrid.hdz.horta@gmail.com; marthajimenezga@gmail.com \\ * Autor a quien debe ser dirigida la correspondencia
}

Recibido Ene. 29, 2018; Aceptado Abr. 11, 2018; Versión final Jun. 1, 2018, Publicado Oct. 2018

\begin{abstract}
Resumen
El objetivo de esta investigación es conocer el impacto del uso de las aulas virtuales en la educación superior. Se realizó una investigación descriptiva y cuantitativa en el Instituto Politécnico Nacional (IPN) en México, dentro de la Unidad Profesional Interdisciplinaria de Ingeniería y Ciencias Sociales y Administrativas (UPIICSA). Se realizó una encuesta a una muestra aleatoria de 428 alumnos. Los resultados muestran que los alumnos de UPIICSA no usan las aulas virtuales, el promedio académico es de 7.98 y $36 \%$ de los estudiantes tienen materias reprobadas. Se concluye que las aulas virtuales no son usadas por los estudiantes, pues estas no han sido implementadas por los profesores. Sin embargo, si se hiciera uso de estas tecnologías, los alumnos podrían incrementar su rendimiento académico aprovechando los elementos y recursos que ofrece esta tecnología para incrementar su rendimiento académico.
\end{abstract}

Palabras clave: aulas virtuales; educación superior; rendimiento académico; estudiantes; profesores

\section{Digital Classrooms in Higher Education: The Case of Mexico}

\begin{abstract}
The objective of this research is to know the impact of the use of virtual classrooms in higher education. A descriptive and quantitative research was carried out at the National Polytechnic Institute (IPN) in Mexico, within the Interdisciplinary Professional Unit of Engineering and Social and Administrative Sciences (UPIICSA). A random sample of 428 students was surveyed. The results show that UPIICSA students do not use virtual classrooms, the academic average is 7.98 and $36 \%$ of students have failed subjects. It is concluded that virtual classrooms are not used by students, as they have not been implemented by teachers. However, if these technologies were used, students could increase their academic performance by taking advantage of the elements and resources offered by this technology. to increase their academic performance.
\end{abstract}

Keywords: virtual classrooms; higher education; academic performance; students; teachers 


\section{INTRODUCCIÓN}

Las Tecnologías de Información y Comunicación (TIC), generan hoy en día, una revolución de mayor impacto en comparación a la que generó la escritura, la industrialización o la imprenta, esto debido a que ha constituido una sociedad del conocimiento a lo largo del mundo con el fin de acceder a la información y poder comunicarse mejor mediante las acciones a distancia, las acciones de red y las acciones asíncronas, como ejemplo de ello tenemos al comercio electrónico, chat, compra y venta de acciones por internet (Echeverría, 2008). Como resultado de estos avances tecnológicos, diversos sectores de la sociedad han ido evolucionando tal como la educación. Cabe señalar que la Organización de las Naciones Unidas para la Educación, la Ciencia y la Cultura (UNESCO) expone que el uso de las TIC y la telefonía móvil puede impulsar y promover entornos de aprendizaje más eficaces (UNESCO, 2015).

El uso de técnicas del aprendizaje en línea en la educación superior es cada vez más frecuente, en algunas instituciones, el este aprendizaje ha reemplazado completamente a los métodos de enseñanza tradicionales, mientras que en otros complementa los cursos clásicos (Umek et al., 2015). La actual tendencia de la aplicación de las TIC para la educación es el aprendizaje en línea, por lo que es necesario tener algún dispositivo digital como: computadora de escritorio, computadora portátil, tableta y teléfono inteligente; los cuales están destinados para apoyar el aprendizaje. Dentro de las características que ofrece esta tecnología podemos encontrar: guardar y transmitir lecciones en formato electrónico, uso del almacenamiento en la nube, uso de contenido multimedia como videos, fotos, texto, posible existencia de un instructor (Clark y Mayer, 2016). Algunas formas de aulas virtuales que podemos encontrar son: foros, videos, aulas virtuales (cursos en tiempo real) y aulas virtuales. Estos entornos favorecen en la interacción social debido a que tienen un esquema de aprendizaje colaborativo entre estudiantes, docentes y la comunidad (Avello Martínez y Duart, 2016).

Por otra parte las "Aulas virtuales" o también llamadas entornos virtuales de aprendizaje, se consideran como un ambiente de aprendizaje y enseñanza, localizado con un sistema de comunicación mediante una computadora, que tienen la ventaja de ya no estar construida de ladrillos y tablas, sino con espacios de trabajo y plataformas que están implementados mediante software (Hiltz, 1994). La integración de las aulas virtuales en la educación, tiene diversos beneficios para el trabajo pedagógico las cuales son: el incremento de la motivación, la adecuación de ritmos de aprendizaje, el almacenamiento digital de recursos y la diversificación de actividades de aprendizaje (Vidal, Llanusa, Diego, y Vialart, 2008).

Existe una clasificación de las aulas virtuales las cuales son: semi-presencial, remota, sincrónica y asíncrona. El aula digital semi-presencial, funciona bien como complemento de una clase presencial; los entornos de aprendizaje remotos apoyan a la educación a distancia; con los servicios sincrónicos se puede recibir o enviar un mensaje, sin embargo, es necesario que dos o más personas estén presentes en el mismo, como ejemplo podemos nombrar las videoconferencias o chat, los servicios asincrónicos son aquellos que permiten la transmisión de un mensaje entre el emisor y el receptor sin que tengan que coincidir para interactuar en el mismo instante, algunos ejemplos de este servicio son: página web, e-mail y foros de discusión (Lara, 2002). Para el uso de las aulas virtuales, es necesario la integración del profesor para que sirva como orientador y supervisor, además, que se encargue de tareas como la gestión y entrega de actividades (Bustos y Salvador, 2010; Vidal et al., 2008). Del mismo modo la actitud del docente es importante y es un elemento central para el buen aprovechamiento de las TIC dentro del aula, pues quienes tienen disposición abierta al trabajo con las TIC hacen mejor aprovechamiento de la infraestructura tecnológica, en comparación de aquellos quienes no están a favor de la integración tecnológica (Padilla y López de la Madrid, 2013).

Los docentes a menudo no exploran los entornos virtuales de aprendizaje en todo su potencial y solo adoptan un conjunto limitado de herramientas disponibles, asimismo algunos profesores comparten sus cursos entre ellos para fortalecer los entornos virtuales (Derboven et al., 2017). Sin embargo, la enseñanza teórica puede complementarse con simulaciones digitales, lo que es una opción en la universidad para reforzar los conocimientos teóricos y experimentales (Deng et al., 2018). Las estrategias didácticas con material multimedia se pueden usar para proporcionar un desarrollo profesional a los profesores, para que tengan mejores prácticas efectivas de enseñanza (Kennedy et al., 2017). Las tecnologías basadas en las redes sociales como Facebook, Twitter e Instagram facilitan el debate en el aula virtual, pero los profesores pueden resistirse a estas tecnologías por temor a perder la capacidad de guiar el debate; esto favorece a las aulas virtuales y apoya en el rendimiento académico (Ersoy-Babula et al., 2018).

Los estudiantes de la Universidad de Salamanca usan los buscadores en la red, los portales de video en línea, las bibliotecas virtuales y algunos buscadores web; asimismo valoran la ayuda y la importancia de las TIC en su proceso de aprendizaje y consideran positivamente las TIC como herramientas relevantes, por otro lado, dependiendo de las áreas de conocimiento existen diferencias en el uso de las TIC, pues los estudiantes pertenecientes al área de Ciencias de la Salud presentan un alto nivel de preferencia en el uso de TIC mientras que el resto de los alumnos vinculados Arte y Humanidades, Ciencias, Ciencias Sociales y Jurídicas e Ingeniería y áreas de Arquitectura muestran un bajo nivel de preferencia en el uso de TIC (Vega-Hernández et al., 2018). 
Con respecto a la eficiencia del uso de aulas virtuales, diversas investigaciones indican usar la tecnología, pues dicen que es igual de efectivo que las clases en modalidad escolarizada. A manera de ejemplo mencionaremos el trabajo realizado por Popescu y Navarro (2005), en la Facultad de Medicina de la Universidad Nacional del Nordeste, Argentina; pues ellos Indicaron un incremento en el promedio académico de los estudiantes de medicina del 8.39 \pm 2.14 al 9.76 \pm 2.8 . Así mismo una investigación realizada por Viada et al., (2011) concluye que el aula virtual de estadística desarrollado por ellos, puede ser utilizado como sustituto de la práctica real en la enseñanza de la estadística en carreras universitarias, que no contienen en su plan de estudios prácticas de aula con sistemas estadísticos, tales como biología, química, estomatología y Medicina (Viada González et al., 2011). A pesar de que algunos autores concluyen que un aula virtual puede ser un sustituto de un aula tradicional, otros como Oproiu (2015), exponen que los salones digitales no pueden reemplazar por completo la educación tradicional.

La investigación realizada por Cakiroglu (2014), evalúa la calidad del aprendizaje a distancia en la educación superior considerando los siete principios de buenas prácticas (SPGP), sus resultados muestran que las perspectivas con respecto al aprendizaje en general eran "Muy satisfactorio", mientras que la subsección de aprendizaje activo se evaluó como "satisfactoria", además la dimensión de "enseñanza" de SPGP se consideró "altamente satisfactoria". Del mismo modo, el estudio realizado por Herrera Mosquera (2017), muestra que los estudiantes se sienten motivados hacia el uso de un entorno virtual de aprendizaje y sugieren que todos los docentes deberían usarlo en sus clases. Por otro lado, las aulas virtuales fomentan la revitalización del aula desocupada con actividades fuera de clase. Además, las comunidades fuertes y dispersas deben coexistir para mantener y revitalizar el aula desocupada de manera sostenible (Yamashita, 2017). Los laboratorios virtuales también son utilizados para presentar nuevas formas de enseñar sistemas complejos de TIC, lo cual favorece al aumento del conocimiento (Kankare y Kasurinen, 2017). Para finalizar, el uso de un aula virtual contribuye a corregir las deficiencias de aprendizaje reconocidas por los estudiantes, sin embargo es necesario de una correcta gestión del tiempo y habilidad para llevar a cabo un estudio independiente (Lamas et al., 2010).

Por lo que se refiere a incluir el uso de las TIC en la educación según lo planteado por la UNESCO, además de que los autores mencionados previamente sugieren el uso de las TIC para tener un mejor aprendizaje, el fenómeno de estudio de esta investigación es analizar las aulas virtuales que son utilizadas en la universidad y su relación con el rendimiento académico. Por lo que el objetivo de esta investigación es analizar la información proporcionada por los alumnos para explicar de forma descriptiva el impacto del uso de las aulas virtuales en la educación superior en una universidad en México. Por consiguiente, surgen las preguntas de investigación ¿Los alumnos usan las aulas virtuales como Moodle, Schoology, Edmodo?, ¿Existe alguna relación entre los alumnos que tienen beca y los que no, en el uso de las aulas virtuales?, ¿Se tiene relación entre en número de materias reprobadas con las aulas virtuales?; asimismo se tiene como hipótesis planteada que en una universidad pública de México no se usan las aulas virtuales, para esto se consideró como variable dependiente la frecuencia de uso de las aulas virtuales, y como variables independientes la cantidad de alumnos que usan las aulas virtuales (Moodle, Schoology, Edmodo), el número de alumnos con beca y las materias reprobadas.

\section{OTROS ANTECEDENTES}

Hay otros antecedentes que es necesario describir para ubicar el tema en el contexto de interés en esta discusión: aspectos sobre la Educación en México, el Uso de las TIC en México, el uso de las plataformas Moodle, Schoology y Edmodo

\section{La Educación en México}

El Plan Nacional de Desarollo 2013-2018 de México indica que el Sistema Educativo Nacional atiende actualmente a 35.2 millones de niños y jóvenes, en la modalidad escolarizada y colaboran alrededor de 1.2 millones de profesores en casi 228 mil escuelas. En los planteles de educación media superior se está atendiendo a 4.4 millones de jóvenes, correspondiendo el $91.3 \%$ a los bachilleratos y $8.7 \%$ a la educación profesional técnica y por cada 100 egresados del bachillerato, 85.9 se inscriben en alguna institución de educación superior. La matrícula de la educación superior es de 3.3 millones de alumnos (Federal, 2013). Con respecto al rezago educativo, la población adulta mayor de 15 años es de 32.3 millones, que no han completado la educación básica, 15 millones de personas mayores de 18 años no han completado el bachillerato, requisito para acceder a mejores oportunidades de trabajo. Esta situación conlleva un alto costo económico, social, y humano, ya que se desaprovecha el recurso más importante del cual goza México hoy en día: una juventud que tiene el potencial de convertirse en el gran motor del desarrollo económico y social del país (Federal, 2013). En definitiva, México debe solventar el rezago educativo y brindar los servicios de las aulas virtuales en todos los niveles educativos tal como hacen las principales ciudades asiáticas como Singapur, Hong Kong, Taiwán y Beijing que invierten continuamente en aulas virtuales para empoderar a los 
líderes escolares para avanzar en la educación escolar en la era digital (Kong et al., 2017); por lo que esta investigación es importante de forma inicial.

\section{Uso de las TIC en México}

Con respecto al uso del internet, los datos proporcionados por la Asociación Mexicana de Internet describe el perfil que tienen las personas quienes acceden al internet y se destaca: 1) la edad de los internautas la cual tiene un rango de 13 a 55 años y representa el 85\% de la muestra; 2) el tiempo diario promedio de conexión es de 7 horas y 14 minutos; 3 ) el lugar donde más frecuencia se hace uso del internet es el hogar seguido de "en cualquier lugar" mediante un dispositivo móvil; 4) el dispositivo más utilizado por los internautas es el Smartphone seguido de la laptop y computadora de sobremesa (AMIPCI, 2016). EIPlan Nacional de Desarrrollo en Mexico propone: 1) crear una red nacional de centros comunitarios de capacitación y educación digital; 2) promover mayor oferta de los servicios de telecomunicaciones; 4) promover la inversión privada en el sector de las TIC; 5) continuar y ampliar la Campaña Nacional de Inclusión Digital; 6) aumentar el uso del Internet mediante el desarrollo de nuevas redes de fibra óptica; 7) desarrollar e implementar la infraestructura espacial de banda ancha incorporando tecnologías satelitales (Federal, 2013). Recapitulando, para enriquecer la calidad de los estudiantes es necesario un plan estratégico de salones digitales que incluyan actividades de aprendizaje interactivo más allá de las que se pueden lograr a través de las interacciones cara a cara en el aula, para que los estudiantes aprendan de manera colaborativa, auto dirigida y personalizada en cualquier momento y en cualquier lugar (Wong et al., 2014). A continuación, se describen algunas plataformas de aulas digitales, en las que se basa esta investigación.

\section{Moodle}

La plataforma Moodle es utilizada en todo el mundo, por ejemplo en Rumanía la utilizan y les ayuda a mejorar el rendimiento académico de los estudiantes y les proporciona más confianza en sí mismos, estudiando y entendiendo mejor los contenidos del curso, además es un soporte para los profesores (Chen et al., 2014 y Oproiu, 2015). A pesar de que Moodle es una plataforma digital muy eficiente, presenta algunos inconvenientes técnicos, por lo cual es conveniente realizar mantenimiento en laboratorios de computación y asignar más apoyo técnico a estos laboratorios para ayudar a los estudiantes a superar los principales problemas que enfrentan al usarlo (Almarabeh et al., 2014). Con la plataforma Moodle se han presentado contenidos personalizados de acuerdo con los antecedentes de los alumnos y los objetivos de aprendizaje, a través de una planeación con inteligencia artificial como un formalismo muy útil para mapear acciones; por lo que es viable utilizar el salón digital (Caputi y Garrido, 2015). Además, un curso puede mejorarse utilizando tecnologías educativas como plataformas de enseñanza basadas en Moodle, pues esto compromete al alumno a mejorar su rendimiento de aprendizaje, lo que a su vez mejora la satisfacción del alumno al asimilar nuevos conceptos y desarrollo de nuevas habilidades para resolver problemas de negocios en el mundo real (Rueda et al., 2017).

\section{Schoology}

Schoology es una plataforma de administración de aprendizaje y redes sociales en línea, que amplía el aprendizaje más allá de las limitaciones del aula, tiene características similares con Facebook, ya que redefinió el sistema de gestión del aprendizaje para ofrecer educación en línea como un esfuerzo colectivo para aumentar el impacto de todos los estudiantes que participan en el proceso educativo, con una interfaz colaborativa flexible, que fortalece la instrucción atractiva y la colaboración de todo el sistema en cualquier dispositivo (Anshari et al., 2015 y Schoology, 2014). Cabe mencionar que en Schoology, el profesor puede compartir notas, asignar tareas, calificar su trabajo, atender la asistencia y brindarles debate en línea; por lo que a los estudiantes les gusta este salón digital, debido a que la aplicación la pueden descargar e instalar en sus teléfonos inteligentes y pueden ver las notas desde cualquier lugar y en cualquier momento que lo deseen (Anshari et al., 2015). Además, la plataforma Schoology incluye una forma sencilla de evaluar el progreso de los estudiantes de forma dinámica y rápida, lo que aumenta su participación y logros (Sarrab et al., 2015 y Vicent et al., 2015). Es por ello que esta plataforma ha sido exitosa dentro de las comunidades estudiantiles donde se ha utilizado, puesto que su accesibilidad y flexibilidad son las características más atractivas que posee esta aula virtual.

\section{Edmodo}

La plataforma Edmodo proporciona: motivación, empoderamiento e innovación, pues los estudiantes están motivados para pasar el tiempo aprendiendo como mejorar su práctica, además se sienten facultados para realizar cambios en su práctica, y creen que pueden adoptar prácticas innovadoras y centradas con sus pares académicos (Trust, 2017). Edmodo tiene la particularidad de crear un espacio virtual donde se da la confianza a los alumnos para dialogar y llevar a cabo las clases programadas, de manera efectiva. Una de las principales 
causas del éxito de Edmodo dentro de las comunidades estudiantiles es que utiliza la Gamificación aplicada al aprendizaje, gracias a la Gamificación los profesores son capaces de otorgar pequeños reconocimientos virtuales a los alumnos, los cuales aumentan los niveles de motivación y compromiso de los alumnos con su propio aprendizaje. Además, los estudiantes experimentan un aprendizaje innovador, activo y profundo en un aula virtual, independientemente de la hora y su ubicación (Wu et al., 2017).

Asimismo. es importante mencionar que la mayoría de los profesores de la universidad de estudio, son profesores de contrato por horas frente a grupo, es decir no tienen descarga académica para realizar actividades complementarias como sería el caso de la implementación de plataformas de aulas virtuales; lo cual conduce a que no hubo oportunidad de aplicar la encuesta a los profesores por falta de tiempo, por lo que se decidió aplicar la encuesta a los alumnos, para poder conocer la implementación y uso de las aulas virtuales.

\section{METODOLOGÍA}

La investigación es cuantitativa y descriptiva, fue realizada en la Ciudad de México, dentro de la Unidad Profesional Interdisciplinaria de Ingeniería y Ciencias Sociales y Administrativas (UPIICSA) perteneciente al Instituto Politécnico Nacional que imparte los siguientes programas académicos: Licenciatura en Administración Industrial; Licenciatura en Ingeniería en Transporte; Licenciatura en Ingeniería en Informática; Licenciatura en Ciencias de la Informática y Licenciatura en Ingeniería Industrial. Se realizó en esta Unidad Académica por ser de tipo multidisciplinaria, pues tiene programas de estudio de las áreas de ingeniería y sociales, por lo cual los resultados encontrados aplican para otras Instituciones de Educación Superior en México.

El instrumento utilizado para la recopilación de información fue una encuesta, que tiene como fin identificar aquellas herramientas basadas en las TIC que apoyen a los estudiantes de licenciatura que actualmente cursan las carreras ofrecidas por la unidad. La encuesta está estructurada por 52 preguntas divididas en 12 secciones, la sección utilizada para el desarrollo de este trabajo fue "Aulas virtuales" la cual contenía las siguientes preguntas: ¿Qué tan seguido usas Moodle?, ¿Qué tan seguido usas Schoology?, ¿Qué tan seguido usas Edmondo?, entre otros datos generales. Cabe añadir que la encuesta contempló variables cuantitativas como el tiempo (días).

El instrumento se aplicó a una muestra aleatoria de 428 alumnos de una población aproximada de 13,000, para el tamaño de la muestra se utilizó el cálculo cuando se conoce el tamaño de la población, para esto se realizó con la fórmula de la ecuación 1.

$$
n=\frac{N * Z_{\alpha}^{2} * p * q}{d^{2}(N-1)+\left(Z_{\alpha}^{2} * p * q\right)}
$$

Donde:

$\mathrm{N}$ = tamaño de la población

$\mathrm{Z}=$ nivel de confianza,

$\mathrm{p}=$ probabilidad de éxito, o proporción esperada

$q=$ probabilidad de fracaso

$\mathrm{d}=$ precisión (Error máximo admisible en términos de proporción)

Para nuestro caso se utilizó un tamaño de población de 13000, con una seguridad del 95\%, una precisión del $3 \%$ y una proporción esperada del $5 \%$. Esto nos dice que se requieren encuestar a no menos de 73 estudiantes para poder tener una seguridad del $95 \%$, sin embargo, se logró encuestar a 423 alumnos. Para la inclusión de los sujetos de estudio, se decidió realizar un muestreo aleatorio independiente del semestre o programa de estudios, esto a fin de que la información no fuera sesgada, en cuanto a la validez del instrumento se realizó una prueba de Cron Bach a un piloto de 5 estudiantes, el resultado fue un alfa de Cronbach (0.81). Asimismo, se analizó una correlación de Pearson entre las variables seleccionadas y las que se presentan en este estudio tienen una correlación positiva y alta.

A los alumnos encuestados se les pidió que eligieran el nivel de usabilidad que tienen dichas herramientas. Para el análisis de datos sobre el nivel de usabilidad de las aulas virtuales, se hizo uso de una escala numérica que va del 0 al 7 , donde 0 es nunca, 2 es 2 veces por semana, 3 es 3 veces por semana, 5 es 5 veces por semana y 7 es diariamente. 


\section{RESULTADOS}

A continuación, se muestran los resultados de la aplicación de la encuesta en la sección "aulas virtuales". En la Fig. 1 se muestra el número de alumnos que usan el aula virtual de Moodle, el eje de la ordenada de la gráfica representa el número de alumnos y el eje de la abscisa, muestra el número de días a la semana que hacen uso de esa herramienta.

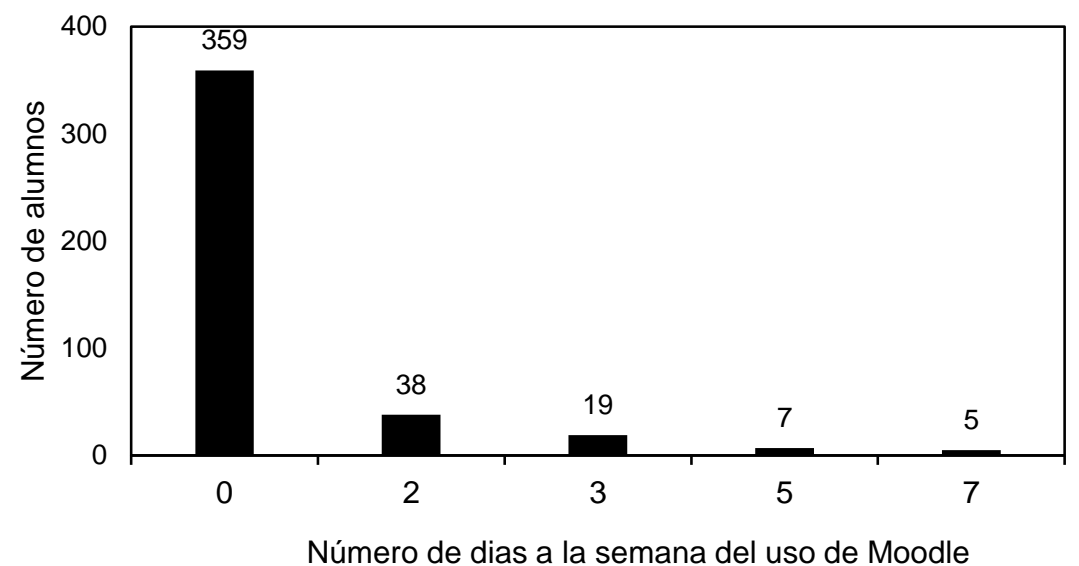

Fig. 1: Número de alumnos que usan el aula virtual Moodle.

En la Fig. 2 se puede observar el número de alumnos que hacen uso del entorno virtual de aprendizaje Schoology, de igual manera que la Fig. 1, el eje de la ordenada de la gráfica representa el número de alumnos, mientras que el eje de la abscisa muestra el número de días a la semana que hacen uso de esa herramienta.

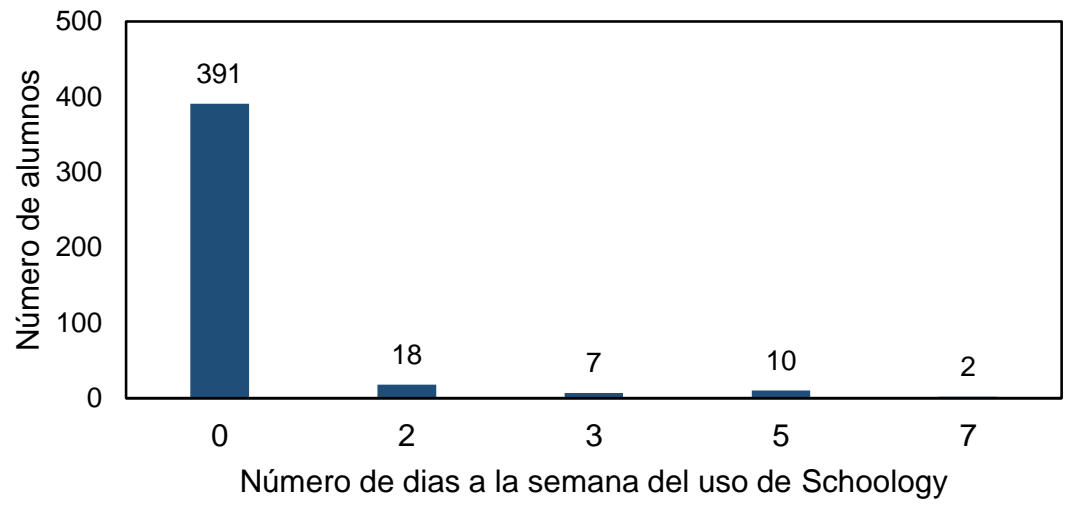

Fig. 2: Número de alumnos que usan el aula virtual Schoology.

En la Fig. 3 se puede observar el número de alumnos que hacen uso del aula virtual Edmodo, de igual manera que la Fig. 1 y 2 , el eje de la ordenada de la gráfica representa el número de alumnos, mientras que el eje de la abscisa muestra el número de días a la semana que hacen uso de dicha herramienta.

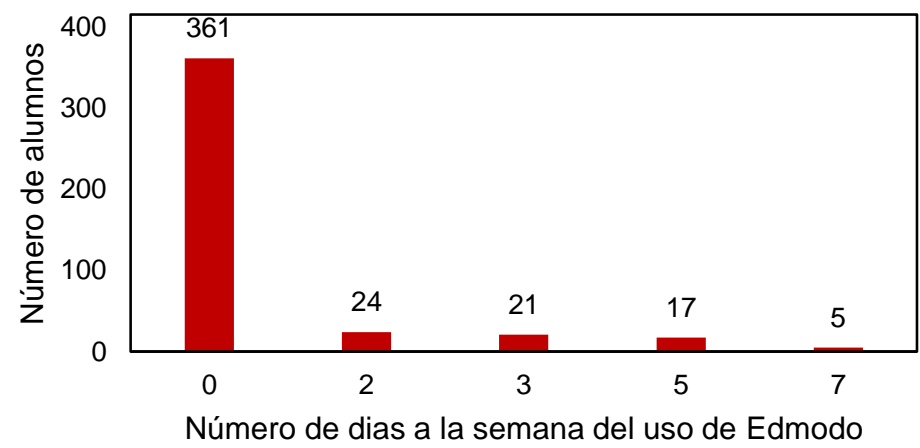

Fig. 3: Número de alumnos que usan el aula virtual Edmodo. 
En la Fig. 4, se muestran el número de alumnos que cuentan con beca, así como también el número de alumnos que no cuentan con beca. El eje de la ordenada de la gráfica representa el número de alumnos, mientras que el eje de la abscisa muestra la situación en la que se encuentra el alumno, con o sin beca.

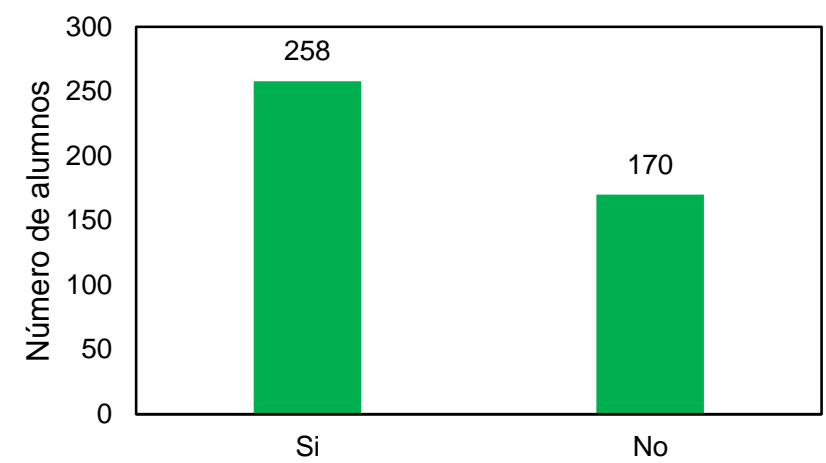

Fig. 4: Número de alumnos que cuentan con beca en la Unidad Profesional Interdisciplinaria de Ingeniería y Ciencias Sociales y Administrativas (UPIICSA).

En la Fig. 5 se presenta la cantidad de materias reprobadas que tienen los alumnos de UPIICSA. El eje de la ordenada de la gráfica representa el número de alumnos, el eje de la abscisa muestra la cantidad de materias reprobadas.

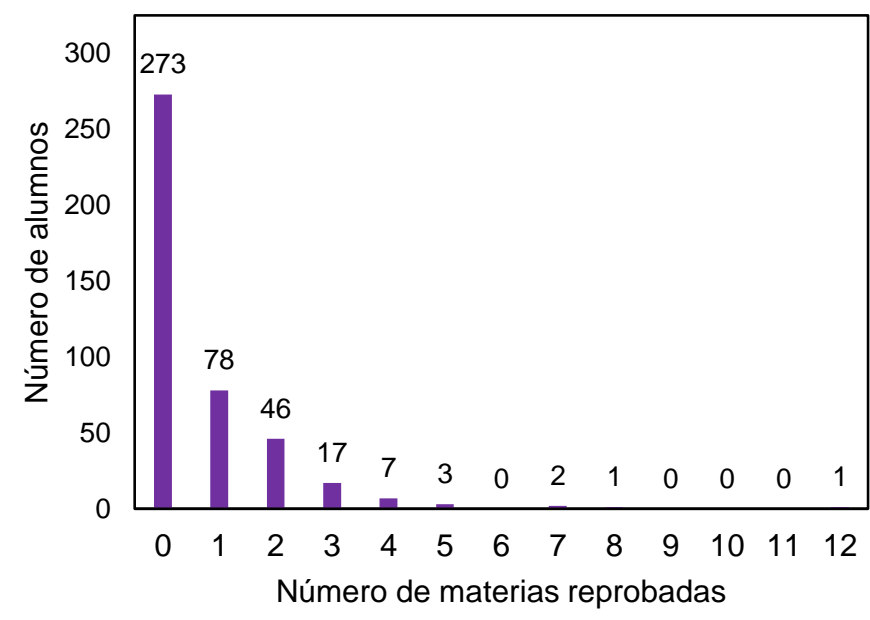

Fig. 5: Numero de materias reprobadas que tienen los alumnos en la Unidad Profesional Interdisciplinaria de Ingeniería y Ciencias Sociales y Administrativas (UPIICSA).

En la Fig. 6 se presenta la cantidad de alumnos encuestados por género, la mayoría son del género masculino. Asimismo, en la Fig. 7 se tiene la distribución de alumnos por programa de estudios y se percibe que la mayoría es de Ingeniería Industrial y de Administración Industrial. Por otro lado, en la Fig. 8 se presenta el promedio de calificaciones (Rendimiento académico de los alumnos) y la media es de 7.98 , así como la división por cuartiles y datos atípicos.

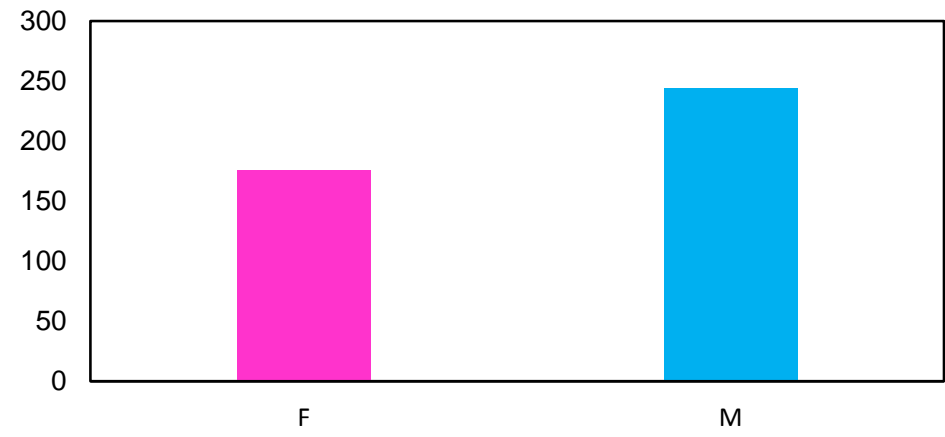

Fig. 6: Alumnos encuestados por género ( $F=$ Femenino, $M=$ Masculino) 


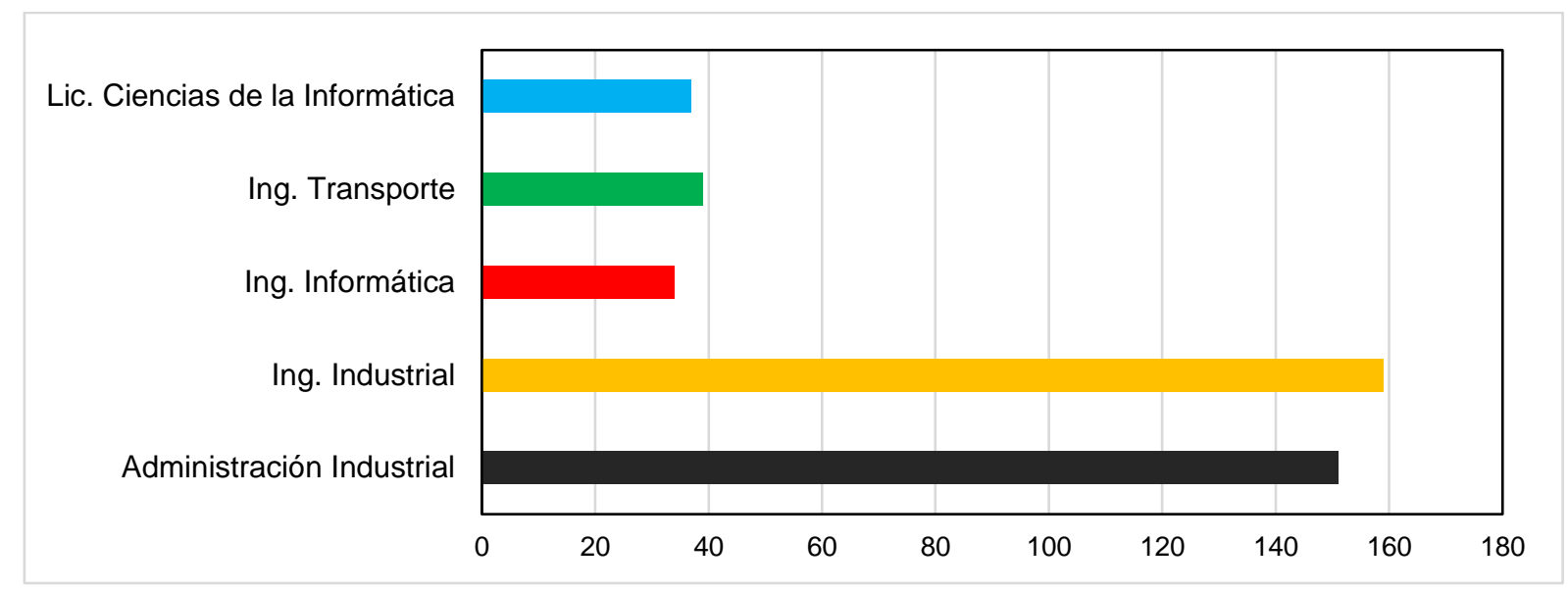

Fig. 7: Alumnos encuestados por programa de estudios.

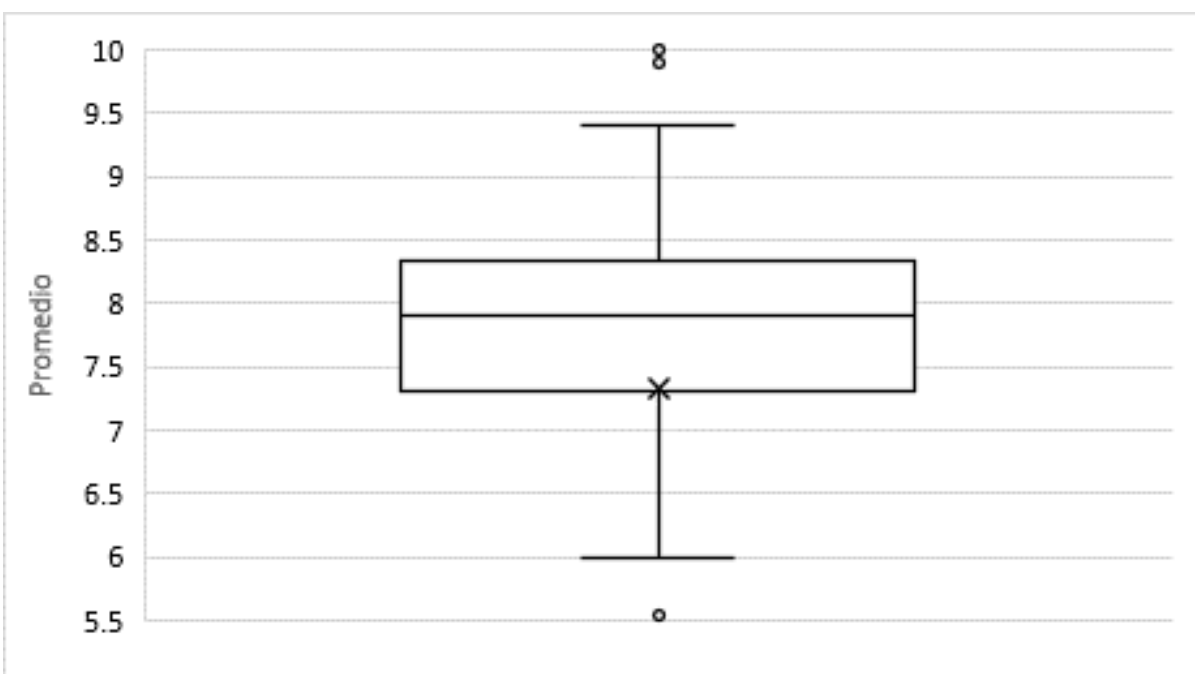

Fig. 8: Promedio de calificaciones de alumnos

\section{DISCUSIONES}

En esta investigación se tiene como supuesto de que los docentes no implementan las aulas virtuales, esto debido a que los profesores carecen de tiempo o no tienen las habilidades necesarias; por lo que se coincide con Dervoben et al. (2017), Kennedy et al. (2017), Deng et al. (2018) y Ersoy-Babula et al. (2018); por tal razón los alumnos no utilizan las aulas virtuales, esto quedo confirmado en los resultados de las Fig. 1,2 y 3 , pues nos muestra que los alumnos no usan las aulas virtuales; esto coincide con los alumnos de Ciencias Sociales y Jurídicas e Ingeniería y áreas de Arquitectura de la Universidad de Salamanca, lo cual presentaron Vega et al. (2018); esto hace suponer que es por el tipo de área del conocimiento, pues son bastante similar, y además esto se confirma en la Fig. 6, pues los hombres son los que estudian ingeniería industrial y fueron la mayoría encuestados y se percibe que deben de incrementar su rendimiento académico, pues el promedio es de 7.96, por lo cual se debería de ver la forma de usar las aulas virtuales.

De acuerdo con los resultados de la investigación, se puede afirmar que los estudiantes de UPIICSA no hacen uso de las aulas virtuales para su educación, siendo esta situación contraria a lo sugerido por la UNESCO (2015), además teniendo en cuenta los resultados de las Fig. 1, 2 y 3 se aprecia que más del $90 \%$ de los alumnos no usan las aulas virtuales Schoology, Modle y Edmodo, a pesar de que se ha demostrado su efectividad justo como lo exponen diversos autores (Almarabeh et al. 2014; Schoology, 2014; Anshari et al., 2015; Caputi y Garrido, 2015; Oman, 2015; Trust, 2017 y Vicent et al., 2015), además de que revitalizan el aula desocupada (Yamashita, 2017), y se pueden utilizar laboratorios virtuales (Kankare y Kasurinen, 2017). En consecuencia, no usan las aulas virtuales, debido a que los profesores no las implementan. Cabe mencionar que Schoology, Modle y Edmodo contienen servicios sincrónicos (Videoconferencia, Chat) y servicios asíncronos (Página web, E-mail: el correo electrónico, Foros de discusión), que ayudarían mejor al estudiante como complemento de una clase presencial. Lo cual hace suponer que en México en las escuelas públicas no se están utilizando estas aulas virtuales. 
Respecto al promedio académico de los estudiantes se puede afirmar que el uso de las aulas virtuales puede ser una oportunidad de mejora en el rendimiento académico de los alumnos, ya que este podría aumentar si se hace uso de las aulas virtuales, pues esto ya fue comprobado por algunos autores (Popescu y Navarro, 2005; Viada González et al., 2011, Rueda et al., 2017 y Wu et al., 2017). Cabe señalar que las aulas virtuales son de fácil acceso a internet con un gran contenido y que además ofrecen una gran cantidad de herramientas (Lara, 2002, Avello Martínez y Duart, 2016; Clark y Mayer, 2016;). Con todos estos beneficios disponibles que ofrecen las aulas virtuales, los estudiantes pueden aprovechar para generar un autoaprendizaje y en consecuencia tener una educación de mayor calidad permitiendo así elevar su rendimiento académico y fortalecer sus habilidades para ser profesionistas con alto nivel de competitividad, por lo cual se sugiere que se busquen políticas educativas que ayuden a implementar el uso de las aulas virtuales.

Otro punto importante a considerar es el problema del rezago educativo en la población adulta (Federal, 2013), se estima que alrededor que 47.3 millones de personas adultas se encuentran en esta situación. Esto nos da un punto de vista de que este escenario se crea en parte al hecho de que los estudiantes de nivel superior truncan sus estudios al reprobar materias durante su trayectoria académica, pues como se muestra en la Fig. 5 , el $36 \%$ de los estudiantes tienen al menos 1 materia reprobada, en consecuencia, en un futuro podrían abandonar la escuela superior. Debemos hacer notar nuevamente que las aulas virtuales en estos casos también son efectivas ya que justo como menciona Lamas et al. (2010), un aula virtual contribuye a corregir las deficiencias de aprendizaje reconocidas por los estudiantes. Por lo cual se reitera la importancia de las aulas virtuales.

Por lo que se refiere a la disposición tecnológica en México en ámbitos de acceso a internet, se puede afirmar que se cuenta con la infraestructura necesaria (AMIPCI, 2016) y las estrategias llevadas a cabo por el Plan Nacional de Desarrollo 2013-2018 en México. A pesar de ello no se hace uso de las aulas virtuales por las siguientes causas: 1) Falta de profesores que diseñen e implementen los cursos en las plataformas digitales gratis; pues en el caso de UPIICSA, la mayor parte de profesores son contratados por menos de 20 horas y éstas deben ser frente a grupo, además dada la situación de desigualdad económica en México, estos profesores tienen otro empleo para poder aumentar su ingreso, por lo cual no tienen el tiempo necesario para diseñar e implementar cursos, 2) Es necesario tener una suscripción para tener acceso al internet y poder estar en alguna plataforma, en adición a esto hay que tener en cuenta como se muestra en la Fig. 4, que el $60 \%$ de los estudiantes de UPIICSA no cuenta con un apoyo económico adicional como es el caso de una beca y en consecuencia le resultaría más difícil acceder al contenido educativo disponible en internet, aunque algunas plataformas sean gratis; pues cuando se realizan actividades con internet, algunos estudiantes no cuentan con internet para realizar las actividades y tienen que compartir la actividad con algún alumno que tenga datos en su dispositivo móvil, 3) En la UPIICSA tampoco se tienen servidores ni personal que los pueda administrar para que se lleven a cabo las aulas virtuales. En consecuencia, se recomienda que sea el gobierno quien establezca las políticas educativas para la implementación y uso de aulas virtuales para que los estudiantes puedan incrementar su rendimiento académico.

\section{CONCLUSIONES}

Las aulas virtuales no son usadas por los estudiantes, pues estas no han sido implementadas por los profesores, sin embargo, si se hiciera uso de estas tecnologías, los alumnos podrían incrementar su rendimiento académico aprovechando los elementos y recursos que ofrece esta tecnología; además las aulas digitales en la educación superior apoyan a combatir el rezago educativo, por lo tanto, los alumnos de UPIICSA y de otras instituciones con materias reprobadas corren el riesgo de truncar sus estudios, sin embargo pueden corregir sus deficiencias de aprendizaje mediante el uso de aulas virtuales; en consecuencia las escuelas de educación superior deben implementar capacitación sobre el uso de aulas virtuales a profesores y alumnos becarios o de servicio social que apoyen a los profesores para que puedan implementar aulas virtuales en plataformas y servidores gratuitos; esto para formar capital humano de calidad para impulsar el desarrollo productivo del país.

\section{AGRADECIMIENTOS}

Agradecemos las facilidades que fueron otorgadas para la realización del presente trabajo al Instituto Politécnico Nacional - Unidad Profesional Interdisciplinaria de Ingeniería y Ciencias Sociales y Administrativas. De igual manera al Proyecto SIP 20161046 las habilidades informáticas del capital humano como fuente de crecimiento económico.

\section{REFERENCIAS}

Almarabeh, T., H. Mohammad, R. Yousef e Y. K. Majdalawi, The University of Jordan E-Learning Platform: State, Students' Acceptance and Challenges, doi: 10.4236/jsea.2014.712087, J. of Software Engineering and Applications, 7(12), 9991007 (2014) 
AMIPCI, $12^{\circ}$ Estudio sobre los Hábitos de los Usuarios de Internet en México (2016)

Anshari, M., Y. Alas, L.S. Guan, Pervasive Knowledge, Social Networks, and Cloud Computing: E-learning 2.0, doi: 10.12973/eurasia.2015.1360a, EURASIA, J. Math., Sci Tech. Ed., 11(5), 909-921 (2015)

Avello Martínez, R. y J.M. Duart, Nuevas Tendencias de Aprendizaje Colaborativo en E-Learning. Claves para su implementación efectiva, doi: 10.4067/S0718-07052016000100017, Estud. Pedagóg., 42(1), 271-282 (2016)

Bustos, A. y C. Salvador, Los Entornos Virtuales Como Espacios de Enseñanza y Aprendizaje. Una Perspectiva Psicoeducativa para su Caracterización y Análisis, ISSN: 1405-6666, Rev. Mexicana de Investigación Educativa, 15 (44), 163-184 (2010)

Cakiroglu, U., Evaluating Students' Perspectives about Virtual Classrooms with Regard to Seven Principles of Good Practice, ISSN: 2076-3433, S. Afr. J. Educ., 34(2), 1-19 (2014)

Caputi, V. y A. Garrido, Student-Oriented Planning of E-Learning Contents for Moodle, J. Netw. Comput. Appl, doi: 10.1016/j.jnca.2015.04.001, 53, 115-127 (2015)

Chen, P. C., T. S. Lan, S. C. Chiu e Y.H. Lan, A Study of Investigating the Learning Effectiveness of Applying the MOODLE E-Learning in Taiwan's Elementary School, doi: 10.6138/JIT.2014.15.7.13, J. of Internet Technology, 15, 1191-1194 (2014)

Clark, R.C. y R. E. Mayer, E-Learning and the Science of Instruction: Proven Guidelines for Consumers and Designers of Multimedia Learning, $4^{\mathrm{a}}$ Ed., 30-31, John Wiley y Sons (2016)

Deng, X., G. Zhou, B. Xiao, Z. Zhao, Y. He y C. Chen, Effectiveness Evaluation of Digital Virtual Simulation Application in Teaching of Gross Anatomy, doi:10.1016/j.aanat.2018.02.014, Ann Anatomy, en prensa (2018)

Derboven, J., D. Geerts y D. De Grooff, Appropriating Virtual Learning Environments: A Study of Teacher Tactics, doi: 10.1016/j.jvlc.2017.01.002, Journal of Visual Languages and Computing, 40, 20-35 (2017)

Echeverría, J., Apropiación Social de las Tecnologías de La Información y la Comunicación, ISSN: 1850-0013, Revista Iberoamericana de Ciencia Tecnología y Sociedad, 4(10), 171-182 (2008)

Ersoy-Babula, A. I. y M. Babula, Learning on the Move Business Students' Adaptation of Virtual Learning Environment and Mobile Device Technology, doi:10.1016/j.jime.2018.04.007, The International Journal of Management Education, 16(2), 321-326 (2018)

Federal, P.E., Plan Nacional de Desarrollo 2013-2018 (2013)

Herrera Mosquera, L., Impact of Implementing a Virtual Learning Environment (VLE) in the EFL classroom. Íkala, doi: 10.17533/udea.ikala.v22n03a07, Revista de Lenguaje y Cultura, 22(3), 479-498 (2017)

Hiltz, S. R., The Virtual Classroom: Learning without Limits via Computer Networks, ilustrada, 3-4, Intellect Books, United States (1994)

Kankare, V. y J. Kasurinen, Simternet-Complex Internet Exercise on a Virtual ICT Learning Environment, doi: 10.1016/j.procs.2017.11.194, Procedia Computer Science, 119, 350-358 (2017)

Kennedy, M. J., S. E. Hirsch, W. J. Rodgers, A. Bruce y J. W. Lloyd, Supporting High School Teachers' Implementation of Evidence-Based Classroom Management Practices, doi10.1016/j.tate.2016.12.009, Teach. Teach. Educ. 63, 47-57(2017)

Kong, S. C., C. K. Looi, T. W. Chan y R. Huang, Teacher development in Singapore, Hong Kong, Taiwan, and Beijing for e-Learning in School Education, doi: 10.1007/s40692-016-0062-5, J. of Computers in Education, 4(1), 5-25 (2017)

Lamas, M.L., A. I. Massié, y E. D. Quero, Implementación de un aula virtual bajo la modalidad mixta: The case of Agricultural Chemistry at the Universidad Nacional de Salta, doi: 10.4067/S0718-50062010000400002, Formación Universitaria, 3(4), 3-12 (2010)

Lara, L. R., Análisis de los recursos interactivos en las aulas virtuales, Segundo Congreso Virtual "Integración sin Barreras en el Siglo XXI", 1-3, Argentina, 01 al 30 de noviembre (2002)

Oproiu, G.C., A Study about Using E-learning Platform (Moodle) in University Teaching Process, doi: 10.1016/j.sbspro.2015.02.140, Procedia-Social and Behavioral Sciences, 180, 426-432 (2015)

Padilla, S. y M. López de la Madrid, Competencias Pedagógicas y Función Docente en las Comunidades Virtuales de Aprendizaje, doi: 10.4067/S0718-07052013000300008, Estudios Pedagógicos (Valdivia), 39 (Especial), 103-119 (2013)

Popescu, B.M. y V. Navarro, Comparación del aprendizaje en internet con la clase convencional en estudiantes de medicina, en Argentina, ISSN: 2014-9832, Educación Médica, 8(4), 204-207 (2005)

Rueda, L., J. Benitez y J. Braojos, From Traditional Education Technologies to Student Satisfaction in Management Education: A theory of the Role of Social Media Applications, doi:10.1016/j.im.2017.06.002, Inform. Management, 54(8), 1059-1071 (2017)

Sarrab, M. y A. S. Hafedh y A. M. Bader, System Quality Characteristics for Selecting Mobile Learning Applications, ISSN: 1302-6488, Turkish Online Journal of Distance Education, 16(4), 18-28 (2015)

Schoology, Mobile Apps Overview, Usage and Workflow, Schoology (2014)

Trust, T., Motivation, Empowerment, and Innovation: Teachers' Beliefs about How Participating in the Edmodo Math Subject Community Shapes Teaching and Learning, doi: 10.1080/15391523.2017.1291317, J. of Research on Technology in Education, 49(1-2), 16-30 (2017) 
Umek, L., A. Aristovnik, N. Tomaževič y D. Keržič, Analysis of Selected Aspects of Students' Performance and Satisfaction in a Moodle-Based E-Learning System Environment, doi: 10.12973/eurasia.2015.1408a, Eurasia J. Math Sci. Technol. Ed, 11(6), 1495-1505 (2015)

UNESCO, La Educación para Todos, 2000-2015: logros y desafíos, 1 a Ed., Organización de las Naciones Unidas para la Educación, la Ciencia y la Cultura, 35-36, París, Francia (2015)

Vega-Hernández, M. C., M. C. Patino-Alonso y M.P. Galindo-Villardón, Multivariate Characterization of University Students Using the ICT for Learning, doi: 10.1016/j.compedu.2018.03.004, Comput. Educ., 121, 124-130 (2018)

Viada González, C., P. Lorenzo Luaces y otros doce autores, Aula virtual de Estadística en soporte multimedia, ISSN: 1684-185, Revista Cubana de Informática Médica, 3(1), 37-46 (2011)

Vicent, L., S. Villagrasa, D. Fonseca y E. Redondo, Virtual learning scenarios for qualitative assessment in higher education 3D arts, doi: 10.3217/jucs-021-08-1086, J. of Universal Computer Science, 21(8), 1086-1105 (2015)

Vidal, L. M., R. S. Llanusa, O. F. Diego y V. N. Vialart, Entornos virtuales de enseñanza-aprendizaje, ISSN: 1561-2902, Educación Médica Superior, 22(1), 1-9 (2008)

Wong, S. Y., W. J. Tee y P. V. Lim, Design Model for Integrating Learning Activity Management System (LAMS), Massive Open Online Courses (MOOC) and Flipped Classroom in Taylor's Integrated Moodle e-Learning System (TIMeS). In S. F. Tang y L. Logonnathan (Eds.), doi: 10.1007/978-981-287-399-6_35, Taylor's $7^{\text {th }}$ Teaching and Learning Conference 2014 Proceedings, 379-387, Singapore (2014)

Wu, Y. C. J., T. Wu, e Y. Li, Impact of Using Classroom Response Systems on Students' Entrepreneurship Learning Experience, doi: 10.1016/j.chb.2017.08.013, Comput. Hum. Behav., 1,12 (2017)

Yamashita, K. y H. Yasueda, Project-Based Learning in Out-Of-Class Activities: Flipped Learning Based On Communities Created in Real And Virtual Spaces, doi:10.1016/j.procs.2017.08.108, Procedia. Computer. Science, 112, 1044-1053 (2017) 
